\title{
Structural Studies of Hydrostatic Extrudates of High Density Polyethylene
}

\author{
Yoji MAEDA, Kazuo NAKAYAma, and Hisaaki KANETSUnA \\ Research Institute for Polymers and Textiles, \\ 1-1-4 Yatabe-Higashi, Tsukuba, Ibaraki 305, Japan
}

(Received April 8, 1982)

\begin{abstract}
A study was made on the superstructure of high density polyethylene extruded hydrostatically at high temperatures. It was found that the X-ray long period for the polyethylene extrudates decreased with increasing extrusion ratio $\left(R_{\mathrm{e}}\right)$. The crystalline and amorphous layer thicknesses of the extrudates with $R_{\mathrm{e}}=4.8$ and 9.0 at $90^{\circ} \mathrm{C}$ were studied by nitric acid etching followed by GPC measurement. It was found that the average lamellar thickness of the crystalline core in the extrudate with $R_{\mathrm{e}}=9.0$ was slightly thicker than that with $R_{\mathrm{e}}=4.8$, while the long period for $R_{\mathrm{e}}=9.0$ was smaller than that for $R_{\mathrm{e}}=4.8$. This fact indicates that both the size of the crystal blocks and the thickness of the interlamellar amorphous layer in the microfibrils decrease remarkably with the extrusion ratio, while the lamellar thickness of the crystalline core in the microfibrils increases slightly. It may be concluded that amorphous chains in the original spherulitic structure are extruded substantially into the interfibrillar region and that these chains in the interfibrillar region form highly oriented bundles.

KEY WORDS High Density Polyethylene /Hydrostatic Extrusion / X-Ray Long Period / Nitric Acid Etching / Gel-Permeation Chromatography / Crystalline Layer Thickness / Amorphous Layer Thickness / Microfibrils / Interfibrillar Amorphous Region /
\end{abstract}

It is well known that wide-angle X-ray diffraction photographs of a high density polyethylene solid extruded with a large deformation ratio exhibit typical $c$-axis orientation patterns. ${ }^{1,2}$ The degree of chain orientation in both the crystalline and amorphous regions in the extrusion direction increases with the extrusion ratio $\left(R_{\mathrm{e}}\right)$. Plastic deformation of fibrous materials can be explained by Peterlin's model $^{3}$ for microfibrillar morphology. In particular, this model can be used to explain the bundling of microfibrils into long, densely packed fibrils. Hydrostatic extrusion of high density polyethylene changes the morphology from spherulitic crystalline lamellae to a highly oriented fiber structure through a complex deformation process involving the destruction of spherulitic crystals, the rotation and sliding of microcrystals, and the rearrangement of fibrils.

We have shown ${ }^{4}$ by wide- and small-angle X-ray scattering (WAXS and SAXS) that hydrostatic extrusion of high density polyethylene both drastically reduces the crystal size and markedly changes the orientation of microcrystals through a change in the fine structure of crystals. The mean dimension $l_{020}$ of the crystal normal to the plane (020), calculated from the WAXS line broadening data, decreases monotonously with increasing extrusion ratio up to about $R_{\mathrm{e}}=4.0$ and thereafter slightly with the extrusion ratio. For example, in the extrusion at $90^{\circ} \mathrm{C}$, $l_{020}$ diminished from 29.0 to $14.0 \mathrm{~nm}$ at $R_{\mathrm{e}}=9.0$ and the WAXS photograph showed the highest degree of orientation. The $a$-axis became aligned perpendicularly to the extrusion direction, while the $b$ axis was well aligned transversely. Further, closely packed thin fibrils of diameters of about $30 \mathrm{~nm}$ were observed by electron microscope on the fractured surface of polyethylene prepared with the ram extrusion. ${ }^{1}$

Nitric acid etching has been widely used for structural investigations of polyethylene, both in bulk and as single crystals. A GPC examination of etched material provides quantitative information on the mechanism of oxidative reactions and the original structure of the polymer. In a previous 
paper, ${ }^{5}$ we applied this procedure to solid-state extrudates of isotactic polypropylene and studied the superstructure of the polymer extruded hydrostatically with $R_{\mathrm{e}}=5.2$ and 6.5 at $150^{\circ} \mathrm{C}$. A series of studies on the superstructure of solid-state extrudates of polymers has been carried out by applying this procedure. In the present study, we report experimental results obtained on high density polyethylene extruded hydrostatically with $R_{\mathrm{e}}=4.8$ and 9.0 at $90^{\circ} \mathrm{C}$ and propose a morphological model for the superstructure of polyethylene extruded at high temperatures.

\section{EXPERIMENTAL}

\section{Sample Preparation}

The resin used in the present study was a commerical rod of high density polyethylene, Hizex 5100B, supplied by Mitsui Petrochemical Co., Ltd. The weight-average molecular weight $M_{w}$ and the weight- to number-average molecular weight ratio $M_{w} / M_{n}$, determined by gel-permeation chromatography, were $1.27 \times 10^{5}$ and 9.4 , respectively. The viscosity-average molecular weight and the melt index reported by the supplier were $8.5 \times 10^{4}$ and 0.25 , respectively. The apparatus and the details of the processes for hydrostatic extrusion are described elsewhere. $^{2}$

A solid cylindrical billet (diameter of up to $6 \mathrm{~mm}$ ) with a nose machined on one head was fitted into the conical entry of the die. Two dies with throats 2.0 and $2.5 \mathrm{~mm}$ wide and $10 \mathrm{~mm}$ long were used. Glycerin was used as the pressure medium. The billet was placed in the extrusion device and heated for about $30 \mathrm{~min}$ at a predetermined temperature, which ranged from room temperature to $110^{\circ} \mathrm{C}$. Extrusion was carried out through a small-angle die (semi-angle die $\alpha, 10^{\circ}$ ) at a constant pressure. The extrusion rates were held at $50-100 \mathrm{~mm} \mathrm{~min}^{-1}$. The extrusion ratio $R_{\mathrm{e}}$ was the cross-sectional area of the original billet, $\pi R^{2}$, relative to the exit, $\pi r^{2}$, of the die, i.e., $R_{\mathrm{e}}=(R / r)^{2}$.

We examined two extrudates prepared with $R_{\mathrm{e}}=$ 4.8 and 9.0 at $90^{\circ} \mathrm{C}$. The crystal form of these extrudates was the usual orthorhombic structure containing a small fraction of monoclinic modification.

\section{Nitric Acid Etching}

Details of the nitric acid etching and GPC measurement are given elsewhere. ${ }^{6}$ The etching of the extrudates was carried out in a sealed flask at $80^{\circ} \mathrm{C}$ using a large excess of Analar-grade fuming nitric acid ( $c a .94 \%$ concn., $1.50 \mathrm{~g} \mathrm{~cm}^{-3}$ ). After a certain period of etching, each extrudate was taken out and washed in distilled water for $12 \mathrm{~h}$, extracted with acetone in a soxhlet extractor for $6 \mathrm{~h}$, and finally dried in vacuo at $40^{\circ} \mathrm{C}$ for $12 \mathrm{~h}$.

\section{Method of Investigation}

Thermal analysis, SAXS, density, and GPC measurements of the polyethylene extrudates both untreated and treated with fuming nitric acid were performed under the same conditions as reported in the previous paper. ${ }^{5}$ Thermal analysis was carried out with a Perkin-Elmer DSC-II differential scanning calorimeter. The melting peak temperature corrected for the thermal lag is taken to be the melting point in each melting endotherm. SAXS patterns were obtained on a Rigaku-Denki diffractometer Rotaunit RU-200 using a vacuum camera. Ni-filtered $\mathrm{Cu}-\mathrm{K} \alpha$ radiation $(45 \mathrm{kV}, 80 \mathrm{~mA})$ and pinhole collimators, 0.3 and $0.2 \mathrm{~mm}$ in diameter, were used. The specimen-to-film distance was $300 \mathrm{~mm}$. Densities were measured by the flotation method using a density-gradient formed by toluene and chlorobenzene at $25^{\circ} \mathrm{C}$. The degree of crystallinity was determined on the basis of the values of Nielson $^{7}$ and Gubler and Kovacs ${ }^{8}$ for the specific volume of the completely amorphous polymer $\left(1.173 \mathrm{~cm}^{3} \mathrm{~g}^{-1}\right)$ and $\mathrm{Swan}^{9}$ for the specific volume of the ideal crystal $\left(1.001 \mathrm{~cm}^{3} \mathrm{~g}^{-1}\right)$. The average molecular weights and molecular weight distributions of the degraded extrudates were determined from GPC curves obtained with a Waters GPC Model 200 , using $o$-dichlorobenzene at $135^{\circ} \mathrm{C}$ as the solvent. The column set and the operating conditions were the same as those used in the previous paper. $^{5}$ Molecular weight calibration for polyethylene was made using three standard samples of polyethylene supplied by the National Bureau of Standards (USA) and two lower molecular weight fractions supplied by Polymer Laboratories, Ltd. The following linear equation was obtained by the least-squares regression,

$$
\log M_{\mathrm{PE}}=-0.290_{3} \times V+12.395_{9}
$$

where $M_{\mathrm{PE}}$ is the weight-average molecular weight of the NBS or PL standard polyethylene fraction, and $V$ is the elution volume at the peak of a GPC 
curve.

It is well known that etched polar molecules remain in the GPC column longer than the corresponding non-degraded chains, but the retention decreases with increasing molecular weight. ${ }^{10}$ It is known ${ }^{11}$ that both the peak width and peak elution volume for polyethylene are scarcely influenced for molecular weights above about 2,000. In this study, therefore, the effect of polar endgroups on the spreading of a GPC curve was neglected.

\section{RESULTS}

\section{Melting Behavior}

Figure 1 shows the dependence of the DSC melting curves on the heating rate for the polyethylene product extruded with $R_{\mathrm{e}}=9.0$ at $90^{\circ} \mathrm{C}$ and the DSC melting curve for the same extrudate degraded with fuming nitric acid for $10 \mathrm{~h}$. These DSC curves show only one sharp melting peak. The melting peak temperature was slightly dependent on the heating rate and was $130.6^{\circ} \mathrm{C}$ at $5^{\circ} \mathrm{C} \mathrm{min}-1$, which was about $4^{\circ} \mathrm{C}$ lower than those for the usual melt-crystallized polyethylenes. The heat of fusion was $44.1 \mathrm{cal} \mathrm{g}^{-1}$ and the degree of crystallinity was

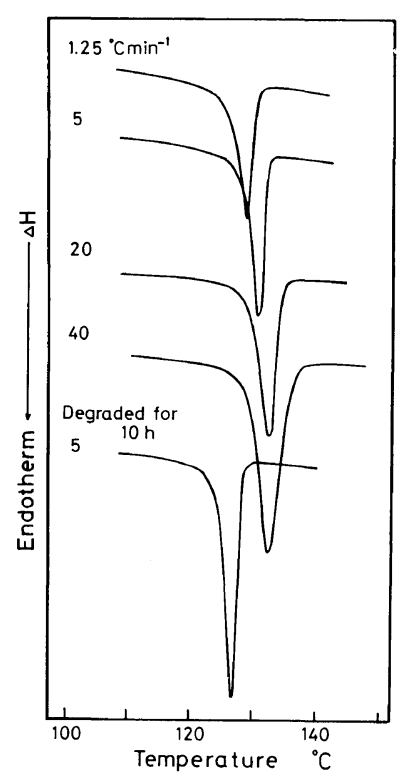

Figure 1. Effect of heating rate on DSC melting curves for polyethylene extruded hydrostatically with $R_{\mathrm{e}}=9.0$ at $90^{\circ} \mathrm{C}$ and for the same product degraded with fuming nitric acid for $10 \mathrm{~h}$. estimated to be $63.5 \%$. The melting curve of the acid-treated sample for $5^{\circ} \mathrm{C} \mathrm{min}^{-1}$ showed a very sharp peak at $126.1^{\circ} \mathrm{C}$, as shown in Figure 1. The melting temperature was $4.5^{\circ} \mathrm{C}$ lower than that of the undegraded sample, but this drop in melting point is usual for acid-treated polyethylene samples. Here, it should be noted that a very sharp melting endotherm was obtained for the degraded sample, which suggests strongly that the extrudate with $R_{\mathrm{e}}=9.0$ has crystals whose lamellar thickness distribution is narrow.

Figure 2 shows the dependence of the melting temperature on the heating rate for the original billet and the two extrudates with $R_{\mathrm{e}}=4.8$ and 9.0 prepared at $90^{\circ} \mathrm{C}$. For the original billet, a small effect of reorganization can be seen at lower heating rates. This effect can also be found to some extent for the extrudate with $R_{\mathrm{e}}=4.8$, but not for the extrudate with $R_{\mathrm{e}}=9.0$. The "equilibrium" melting point for the product with $R_{\mathrm{e}}=9.0$ was easily estimated to be $130.5^{\circ} \mathrm{C}$ by extrapolation to the ordinate in Figure 2. For the original billet and the extrudate with $R_{\mathrm{e}}=4.8$, the "equilibrium" one was taken as the lowest melting temperature in the observed melting peak temperatures at various heating rates, indicating minimal effects of reorganization and superheating. The "equilibrium" melting points so determined were 126.5 and $128.9^{\circ} \mathrm{C}$ for the original billet and the extrudate with $R_{\mathrm{e}}=4.8$, and the corresponding heats of fusion were 30.5 and $41.4 \mathrm{cal}^{-1}$. It should be noted that both the melting points and the heats of fusion for the two extrudates were higher than those for the original billet and that these values increased with the

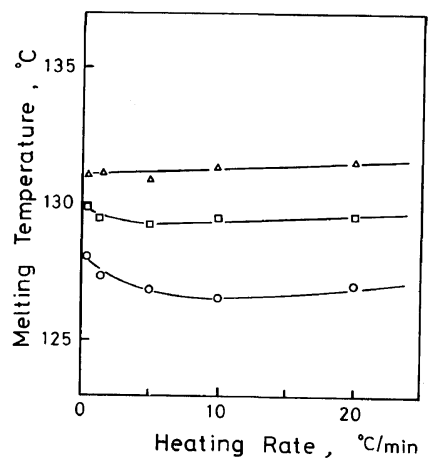

Figure 2. Relation between melting peak temperature and heating rate for the original billet $(O)$ and the extrudates with $R_{\mathrm{e}}=4.8(\square)$ and $9.0(\triangle)$. 


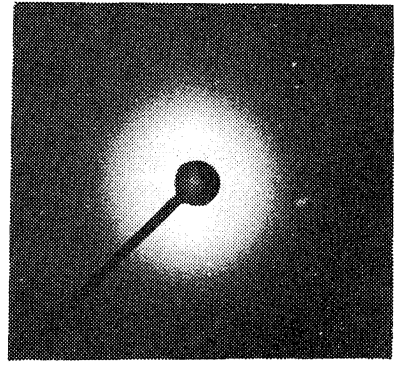

\section{Original}
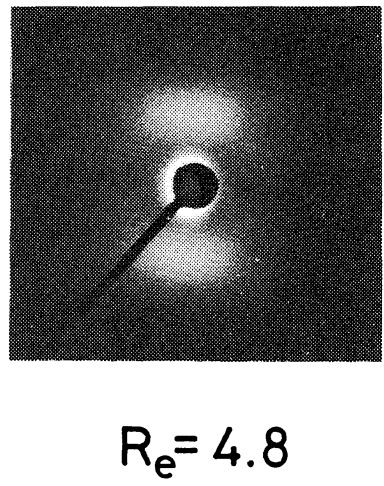

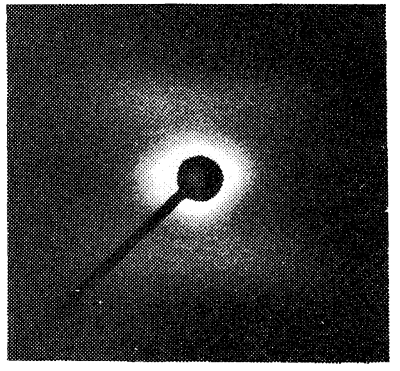

$R_{\mathrm{e}}=9.0$

Figure 3. SAXS photographs of the original billet and the extrudates with $R_{\mathrm{e}}=4.8$ and 9.0 prepared at $90^{\circ} \mathrm{C}$.

extrusion ratio. This suggests an increased degree of crystallinity and thermal stability of high-density polyethylene extrudates.

\section{Small-Angle X-Ray Scattering}

SAXS photographs of the original billet and the extrudates with $R_{\mathrm{e}}=4.8$ and 9.0 are shown in Figure 3. The original billet exhibited a ring with an isotropic intensity distribution, and its WAXS photograph showed a nearly random crystalline orientation. ${ }^{2}$ The SAXS patterns for the extrudates showed two-intensity maxima in the meridian. The line-shaped two point diagram elongated perpendicularly to the meridian with increasing extrusion ratio, while the scattering intensity decreased with the extrusion ratio. The axial long period, indicative of a periodic structure in the polyethylene extrudates, was calculated from SAXS meridional maxima. The values obtained decreased with increasing extrusion ratio and were 23.0, 19.5, and $17.4 \mathrm{~nm}$ for the original billet, the extrudates with $R_{\mathrm{e}}=4.8$ and 9.0 , respectively. This behavior was also observed in the extrusion at $110^{\circ} \mathrm{C}$.

Figure 4 shows the dependence of the long period on the extrusion ratio for the polyethylenes extruded at 30,90 , and $110^{\circ} \mathrm{C}$. This figure clearly shows that the above-mentioned experimental fact is a general trend at extrusion temperatures above about $80^{\circ} \mathrm{C}$. The present finding that the $\mathrm{X}$-ray long period decreases with the extrusion ratio is different from those reported by Clements, Jakeways, and Ward, ${ }^{12}$ according to whom the long period increases slightly with the extrusion ratio. However, in the extrusion at temperatures below $50-70^{\circ} \mathrm{C}$,

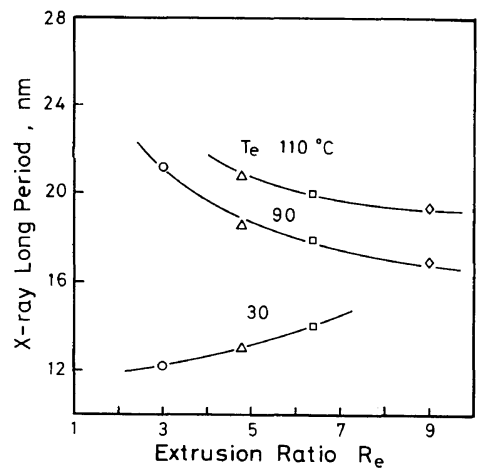

Figure 4. Dependence of the $\mathrm{X}$-ray long period on extrusion ratio for the polyethylenes extruded at 30,90 , and $110^{\circ} \mathrm{C}$.

Table I. Characteristics of polyethylene extruded hydrostatically at $90^{\circ} \mathrm{C}$

\begin{tabular}{cccc}
\hline & \multirow{2}{*}{$\begin{array}{c}\text { Original } \\
\text { billet }\end{array}$} & \multicolumn{2}{c}{ Extrudates } \\
\cline { 3 - 4 } & & $R_{\mathrm{e}}=4.8$ & $R_{\mathrm{e}}=9.0$ \\
\hline$\rho\left(\mathrm{g} \mathrm{cm}^{-3}\right)$ & $0.928_{0}$ & $0.944_{5}$ & $0.948_{0}$ \\
$T_{\mathrm{m}}\left({ }^{\circ} \mathrm{C}\right)$ & 126.5 & 128.9 & 130.5 \\
$\Delta H_{\mathrm{m}}\left(\mathrm{cal} \mathrm{g}^{-1}\right)$ & 30.5 & 41.4 & 44.1 \\
$L_{\mathrm{x}}(\mathrm{nm})$ & 23.0 & 19.5 & 17.0 \\
\hline
\end{tabular}

the axial long period increased with increasing extrusion ratio, as shown in Figure 4.

The "equilibrium" melting points, heats of fusion, densities, and X-ray long periods for our samples are summarized in Table I. 


\section{Nitric Acid Etching}

Figure 5 shows the dependence of the density on the degradation time for the original billet and the two extrudates. Initially, the densities of the three samples increased sharply with degradation time up to about $20 \mathrm{~h}$, and later leveled off to a value of about $1,000 \mathrm{~g} \mathrm{~cm}^{-3}$. Oxidation of polyethylene with fuming nitric acid first causes the molecular chains to break down in the amorphous region between crystallites and then the polar carboxyl and nitro groups become attached to chain ends. The densities of the almost completely etched samples seem to correspond inversely to the densities of the original undegraded samples. The reason for this may be the difference in the content of the cut chain ends or polar end groups in the samples.

The effect of nitric acid etching on the SAXS patterns for the extrudates was noticeable. Figure 6 shows the dependence of the SAXS pattern on the degradation time for the extrudate with $R_{\mathrm{e}}=9.0$ prepared at $90^{\circ} \mathrm{C}$. The scattering intensity of the line-shaped two-point diagram increases during the initial $4 \mathrm{~h}$, then decreases rapidly, finally disappearing at degradation times longer than $10-20 \mathrm{~h}$. In the later stages of degradation, only the streaks can be observed. This may be due to "voids" produced during the nitric acid degradation.

Similar behavior was also observed for the original billet and the extrudate with $R_{\mathrm{e}}=4.8$. This behavior contrasts considerably with that of the hydrostatically extruded polypropylene reported in a previous paper, ${ }^{5}$ in which the SAXS intensity of two-point maxima was found to increase remark-

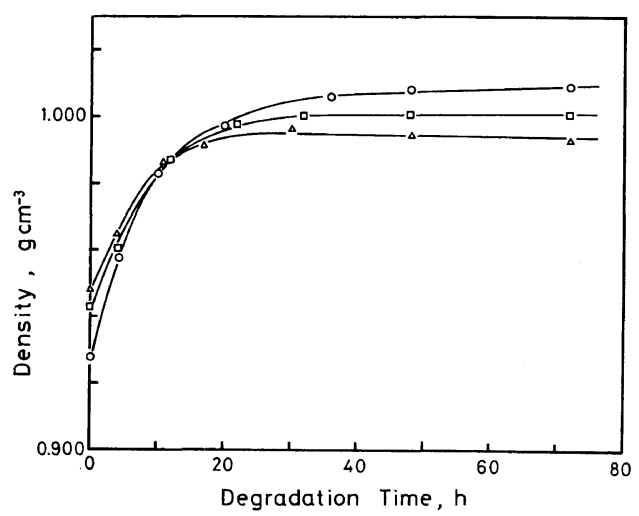

Figure 5. Dependence of density on degradation time for the original billet $(\bigcirc)$ and the extrudates with $R_{\mathrm{e}}=$ $4.8(\square)$ and $9.0(\triangle)$. ably with degradation time.

\section{Gel-Permeation Chromatography}

Figure 7 shows the GPC curves for the original billet degraded with fuming nitric acid for the indicated periods of time. The characteristic feature is that double peaks with a $2: 1$ molecular weight ratio appear after degradation period longer than $20 \mathrm{~h}$. The higher molecular weight peak decreases with degradation time since chain folds are progressively broken, while the lower molecular weight peak is raised. This behavior is usually observed in degradation experiments on folded-chain crystals of polyethylene as in the case of single crystals and bulk-crystallized samples. In the present study, the higher molecular weight peak, indicative of the double chain traverse length of a crystallite, did not disappear even after drastic degradation for $72 \mathrm{~h}$.

Figure 8 shows the GPC curves for the extrudate with $R_{\mathrm{e}}=4.8$ degraded with fuming nitric acid for the periods of time indicated. The GPC patterns changed with degradation time in the same manner as in the original billet, but the higher molecular weight peak was less pronounced than the corresponding peak in Figure 7.

Figure 9 shows the GPC curves for the extrudate with $R_{\mathrm{e}}=9.0$ degraded for the indicated periods of time. The main, broad peak on the GPC curves shifts to a lower molecular weight and at the same time narrows gradually with an increase in degradation time. It should be noted that no double chain traverse peak appears in these curves. However, a small peak can be detected on the GPC curve for $72 \mathrm{~h}$ degradation in the region of molecular weight much higher than the main peak. This peak was reproducible at a molecular weight of about 13,000, and could be distinguished easily from the double chain traverse peaks observed in Figures 7 and 8 . The peak chain lengths of this and the main peak on the curve for $72 \mathrm{~h}$ degradation were estimated to be about 120 and $10.8 \mathrm{~nm}$, respectively.

The lower molecular weight peaks in Figures 79 could be converted to single chain traverse lengths of crystalline cores in the advanced stages of acid etching. When this peak was almost symmetrical, the peak chain length could be converted roughly to the average lamellar thickness of a degraded crystal. In fact, the GPC pattern of the extrudate with $R_{\mathrm{e}}=$ 9.0 became more symmetric about its main peak 


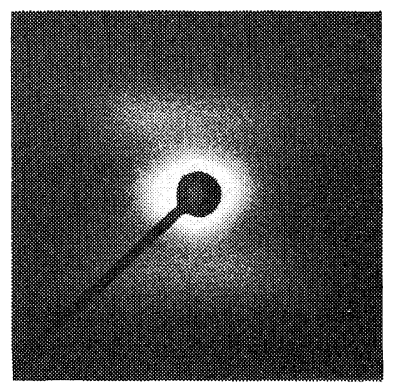

$\mathrm{Oh}$

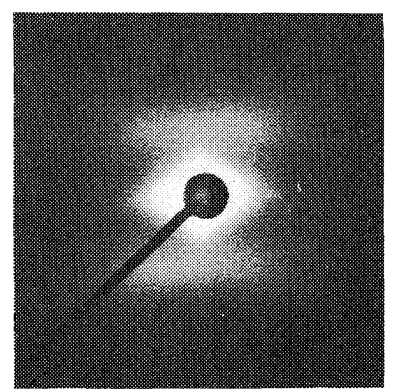

$4 h$

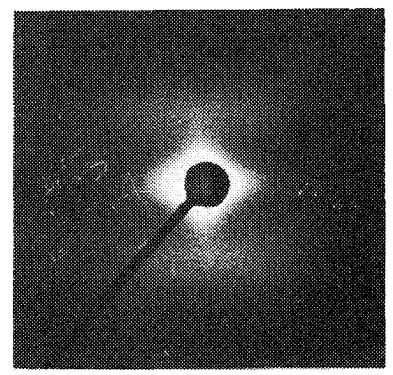

$10 \mathrm{~h}$

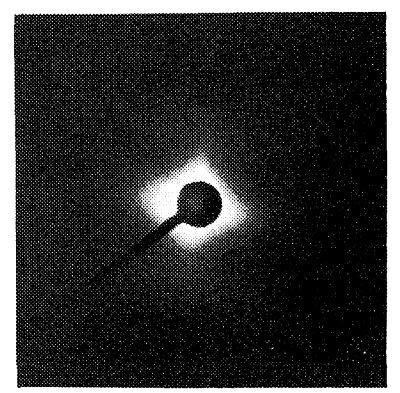

$17 \mathrm{~h}$

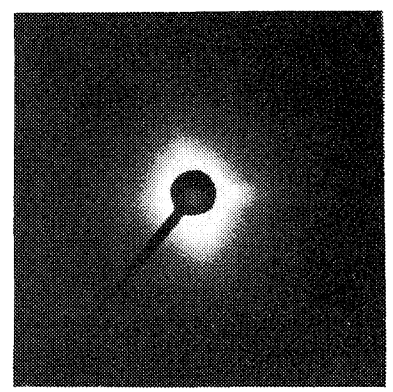

$30 \mathrm{~h}$

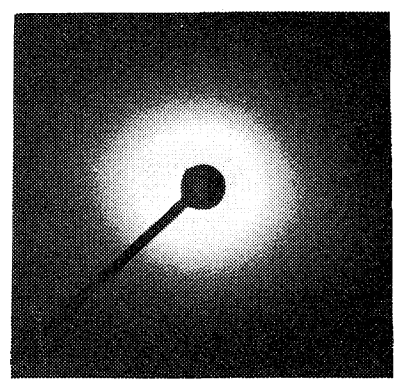

$48 \mathrm{~h}$

Figure 6. Effect of degradation time on the SAXS pattern for the extrudate with $R_{\mathrm{e}}=9.0$.

with an increase in degradation time. It is reasonable to consider that the lower molecular weight peak on the curve for $72 \mathrm{~h}$ degradation corresponds approximately to the lamellar thickness distribution of the crystalline core for the extrudate with $R_{\mathrm{e}}=$ 9.0. If it is assumed that the GPC peak chain length is the average lamellar thickness of a degraded crystal at each stage of degradation, the original lamellar thickness for the undegraded crystal can be estimated by extrapolating the relation between GPC peak chain length and degradation time to $0 \mathrm{~h}$. Figure 10 shows the peak chain length for the GPC lower molecular weight peak at a function of degradation time for the original billet and the two 


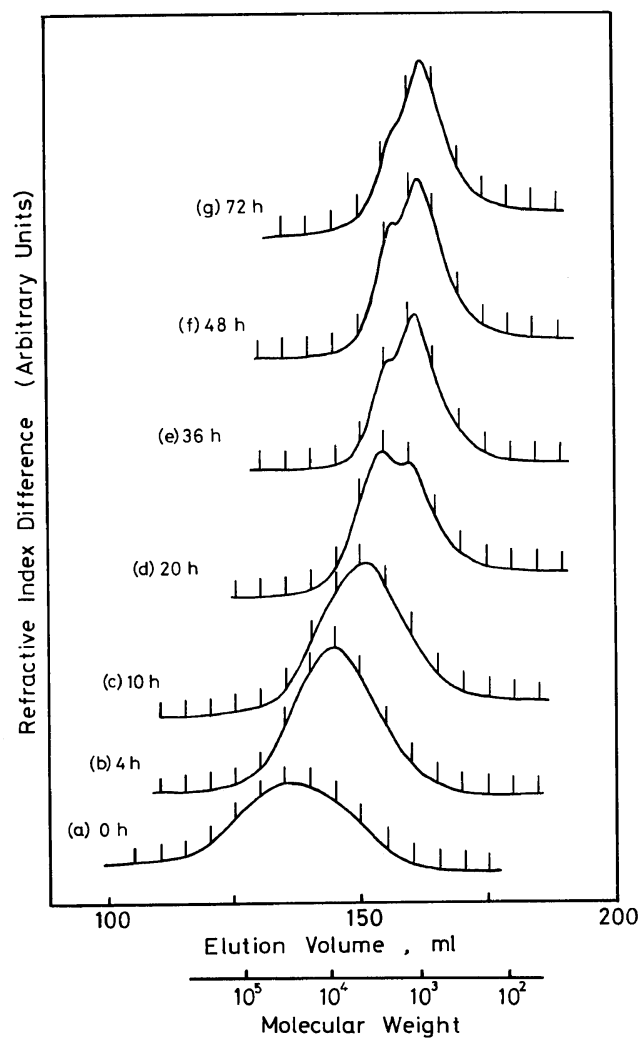

Figure 7. GPC curves of original billet degraded with fuming nitric acid for indicated periods of time.

extrudates. In every case, a rapid decrease in peak chain length was followed by a leveling off in the later stages. The average lamellar thicknesses of the original crystals in the billet and the extrudates with $R_{\mathrm{e}}=4.8$ and 9.0 were found to be $9.0 \pm 1.0$, $10.5 \pm 1.0$, and $12.0 \pm 1.0 \mathrm{~nm}$ by linear extrapolation to the time of $0 \mathrm{~h}$. It is interesting that the average lamellar thickness of the crystallites in the product, extruded at $90^{\circ} \mathrm{C}$, slightly increased unexpectedly with the extrusion ratio, while the axial long priod decreased considerably with the extrusion ratio. Table II gives the values of the axial long period and the extrapolated peak chain length, i.e., the average lamellar thickness of the original crystal in each sample.

A quantitative measure of the molecular weight distribution is the ratio of weight- and numberaverage molecular weights, $M_{w} / M_{n}$. The values of $M_{w} / M_{n}$ for the extrudate with $R_{\mathrm{e}}=9.0$ were 1.8 and 1.5 when estimated from the curves for $48 \mathrm{~h}$ and $72 \mathrm{~h}$

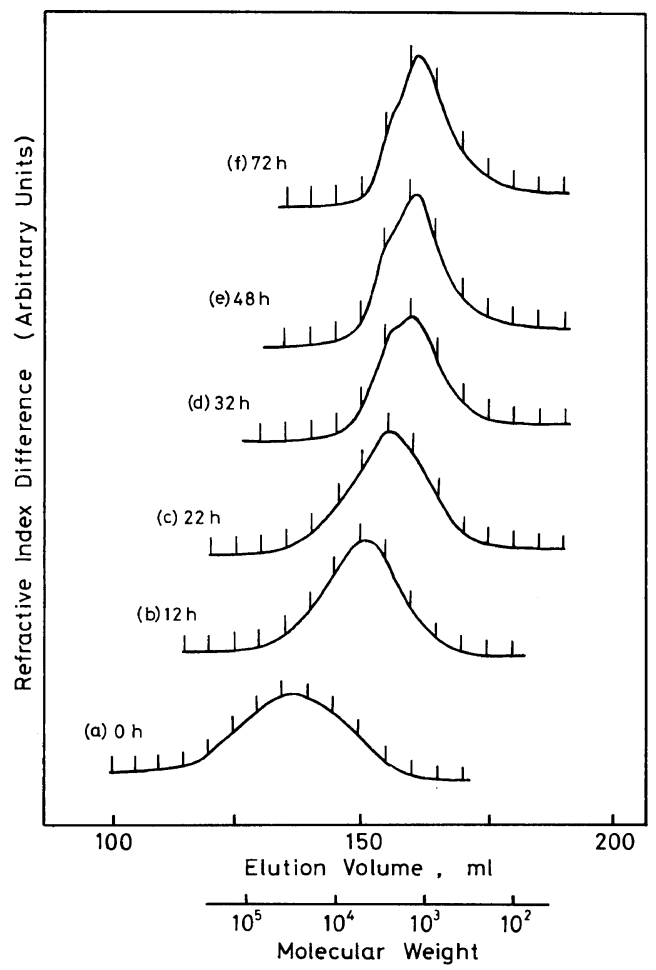

Figure 8. GPC curves of extrudate with $R_{\mathrm{e}}=4.8$ degraded with fuming nitric acid for indicated periods of time.

degradation, respectively. For the extrudate with $R_{\mathrm{e}}=4.8$, this ratio was not estimated because there was no clear separation into double peaks throughout the entire period of degradation. This was due to the uncut folded chains left in the interlamellar crystal surfaces.

\section{DISCUSSION}

In considering the possible structure of hydrostatically extruded polyethylenes, it is necessary to be consistent in two experimentally observed facts. (a) The material retains a periodic structure even at a high extrusion ratio, as indicated by the SAXS patterns. Particularly at extrusions at high temperatures, the X-ray long period decreases with increasing extrusion ratio: (b) The average lamellar thickness of the crystalline core in the chain direction increases slightly with the extrusion ratio, according to the GPC data. The SAXS patterns of the ex- 
trudates indicate that extruded polyethylenes consist, at least in part, of microfibrils with crystalline and amorphous regions arranged in periodic sequences in the direction of extrusion. ${ }^{1,2}$ The experimental results on the SAXS and nitric acid etching plus GPC measurement in the present study show that although the spherulitic crystallites are greatly deformed into small lamellar blocks and highly

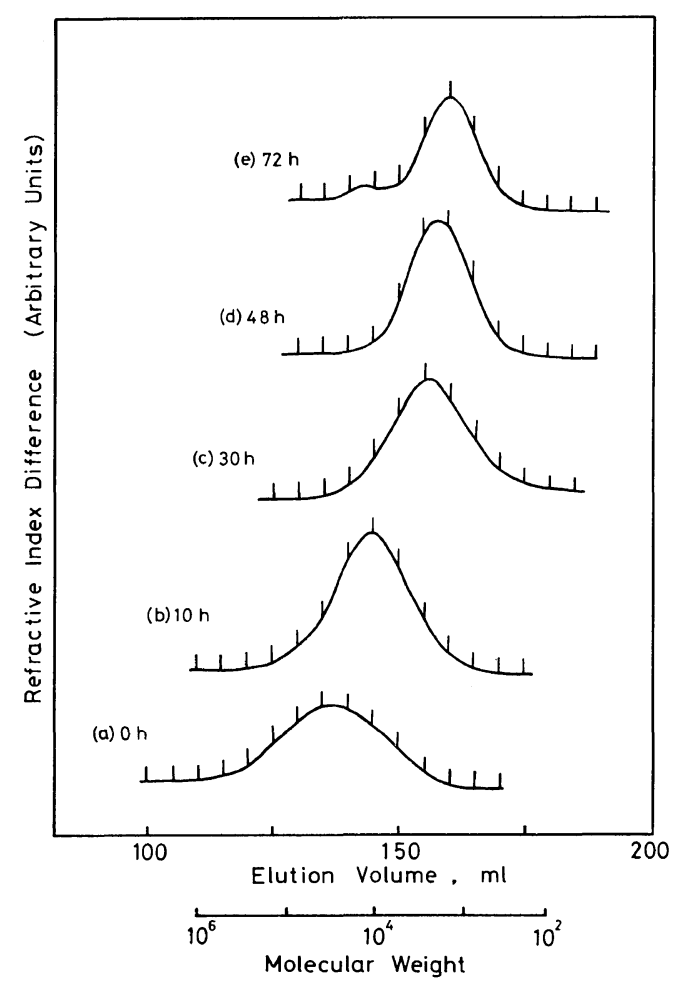

Figure 9. GPC curves of extrudate with $R_{\mathrm{e}}=9.0$ degraded with fuming nitric acid for indicated periods of time. oriented in the extrusion direction during hydrostatic extrusion, the axial lamellar thickness of crystalline core in the microfibrils increases slightly with the extrusion ratio. On the other hand, Clements et at. ${ }^{12}$ reported that both the mean crystal length $\bar{D}_{002}$ in the chain direction and the axial long period increase with increasing extrusion ratio for polyethylene extruded at $100^{\circ} \mathrm{C}$. They indicated that the principal morphological difference between specimens of different extrusion ratios is the increased mean thickness in the $c$-axis direction with an increase in extrusion ratio. But they also suggest that the original folded structure still persists in the specimens but is less clearly defined at high extrusion ratios since the scattered intensity falls substantially as the extrusion ratio increases. The results from the measurement of the X-ray long period for extruded polyethylenes in the present study are clearly at variance with their data, as

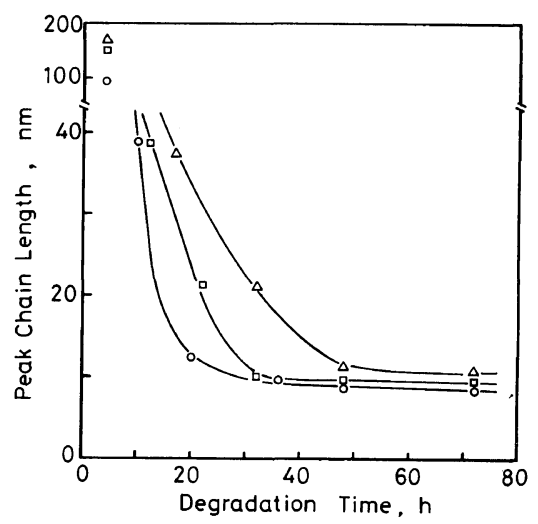

Figure 10. Dependence of the GPC peak chain length on degradation time for the original billet $(O)$ and extrudates with $R_{\mathrm{e}}=4.8(\square)$ and $9.0(\triangle)$.

Table II. Layer thicknesses of crystalline and amorphous regions in polyethylene extruded hydrostatically at $90^{\circ} \mathrm{C}$

\begin{tabular}{|c|c|c|c|}
\hline \multirow[t]{2}{*}{ Sample } & $\begin{array}{l}\text { Long period, } \\
\qquad L_{\mathrm{x}}\end{array}$ & $\begin{array}{l}\text { GPC peak chain length } \\
\text { (crystalline thickness), } \\
L_{\mathrm{c}}\end{array}$ & \multirow{2}{*}{$\begin{array}{l}\text { Amorphous thickness, } \\
\qquad \frac{L_{\mathrm{a}}\left(=L_{\mathrm{x}}-L_{\mathrm{c}}\right)}{\mathrm{nm}}\end{array}$} \\
\hline & $\mathrm{nm}$ & $\mathrm{nm}$ & \\
\hline Original billet & 23.0 & $9.0 \pm 1.0$ & $14.0 \pm 1.0$ \\
\hline \multicolumn{4}{|l|}{ Extrudates } \\
\hline$R_{\mathrm{e}}=4.8$ & 19.5 & $10.5 \pm 1.0$ & $9.0 \pm 1.0$ \\
\hline$R_{\mathrm{e}}=9.0$ & 17.4 & $12.0 \pm 1.0$ & $5.4 \pm 1.0$ \\
\hline
\end{tabular}


evident from Figure 4.

The reason for this is still not clear. However, it may be expected that because of the X-ray long period having a concave trend toward the abscissa in Figure 4, a constant or inversely convex relation may be obtained at extrusion ratios above $R_{\mathrm{e}}=10$, as was the case for hydrostatically extruded polypropylenes in our previous study. ${ }^{5}$

The discrepancy between X-ray long period and GPC peak chain length for extruded polyethylenes might possibly be due to the rough approximation of the thickness of the amorphous layer between crystallites in the microfibrils. Accordingly, the results in the present paper indicate that the decrease in the long period may be attributed to a drastic reduction in the thickness of the amorphous layer in the microfibrils. Table II illustrates clearly that the amorphous layer thickness decreases remarkably with increasing extrusion ratio. The amorphous layer thickness for the extrudate with $R_{\mathrm{e}}=9.0$ is about one third of that of the original billet, i.e., $5.4 \mathrm{~nm}$ to $14 \mathrm{~nm}$. The amorphous layer thickness and lateral crystal dimension for the extrudate with $R_{\mathrm{e}}=4.8$ are intermediate between those for the original billet and the extrudate with $R_{\mathrm{e}}=9.0$. The amorphous layer thickness for the extrudate with $R_{\mathrm{e}}=9.0$ agrees well with Meinel and Peterlin's value $^{13}$ of about $5 \mathrm{~nm}$ for an annealed polyethylene highly drawn $(\lambda=10)$ at $60^{\circ} \mathrm{C}$. It may be considered that the amorphous chains in the original spherulitic structure are extruded substantially into the interfibrillar region. A significant number of amor- phous chains in the extrudate may exist between the microfibrils as highly oriented, chain bundles. In fact, the crystallinity for the product with $R_{\mathrm{e}}=9.0$ increased about $15 \%$. The decrease in SAXS intensity with the extrusion ratio may be due to an increase in the density of the amorphous region resulting from an increase in highly oriented amorphous chains.

Figure 11 shows a schematic structural model for the superstructure of extruded polyethylene, based on the Peterlin's microfibril model, which we propose in order to explain the observations in the present study. The values of the X-ray long period $L_{\mathrm{x}}$, crystalline layer thickness $L_{\mathrm{c}}$, and amorphous layer thickness $L_{\mathrm{a}}$ are presented in Table II. This model has the following important features: (i) Both the crystalline and amorphous regions in the extrudate are highly oriented in the extrusion direction; (ii) The size of the crystal blocks and thickness of the interlamellar amorphous layers in the microfibrils decrease with the extrusion ratio; (iii) The average lamellar thickness of the crystalline core in the microfibrils increases slightly with the extrusion ratio; (iv) The content of folded chains in the extrudate decreases with the extrusion ratio. This suggests an increase in the inter- and intra-fibrillar tie chains in the extrudate; (v) The interfibrillar amorphous region in the extrudate increases with the extrusion ratio. In the advanced stages at much larger extrusion ratios, this model should be replaced by the intercrystallite bridge model for ultrahigh-modulus polyethylene proposed by

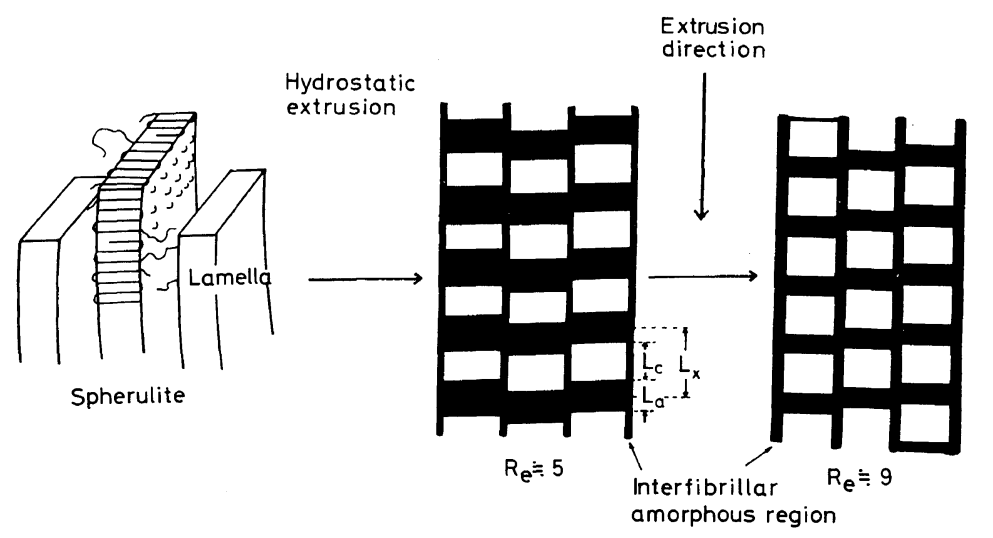

Figure 11. Model of the superstructure in polyethylene extrudates prepared with extrusion ratio up to $R_{\mathrm{e}}=10$ at high temperature. $L_{\mathrm{x}}, L_{\mathrm{c}}$, and $L_{\mathrm{a}}$ denote the X-ray long period, crystalline and amorphous layer thicknesses, respectively. 
Gibson et al. ${ }^{14}$ (modified Takayanagi model ${ }^{15}$ ), which consists of fibrillar stacks of crystallites linked by intercrystalline bridges.

Capaccio and Ward ${ }^{16}$ recently reported an extensive GPC study of two etched ultrahigh-modulus linear polyethylenes; one drawn to a low draw ratio $(\lambda=11)$ at $115^{\circ} \mathrm{C}$ and the other to a high draw ratio $(\lambda=20)$ at $75^{\circ} \mathrm{C}$. They reported that the GPC values of $M_{w} / M_{n}$ in the later stages of etching process are $1.4-1.5$ for the low drawn sample and $2.0-2.1$ for the high drawn one. The value of this ratio for the extrudate with $R_{\mathrm{e}}=9.0$ in the present study is nearly equal to the value of this low drawn sample. The molecular weight distribution for the crystallite thickness of extruded polyethylene may be considered of the same order at nearly the same deformation ratios.

It was possible to detect an additional small GPC peak at a molecular weight higher than the main peak on the GPC curve of the extrudate with $R_{\mathrm{e}}=$ 9.0 degraded for $72 \mathrm{~h}$. This small peak has a much higher molecular weight than the double chain traverse peak as observed on the GPC curves for the original billet and the other extrudate. Weeks, Mori, and Porter ${ }^{17}$ discussed several factors contributing to the breadth of the distribution for ultrahighly drawn polyethylene, such as uncut folds, densely packed, amorphous tie chains, and a wide range of crystal sizes.

The GPC data in the present paper suggest that etching of the extrudates under the employed conditions does not completely cut the molecules in the amorphous region between crytallites in the microfibrils. Fortunately, the situation for the extrudate with $R_{\mathrm{e}}=9.0$ was simpler, since the contribution from only the folds in the later stages of degradation was negligible. Weeks et al. ${ }^{17}$ suggest that ultraoriented strands should contain a large crystal size distribution including a highly acid-resistant, high molecular weight component, probably correspond- ing to extended-chain crystals. However, in this case, it is unlikely that the GPC small peak at a higher molecular weight is associated with the stable crystal including extended-chain crystals, since the extrudate with $R_{\mathrm{e}}=9.0$ showed only one melting peak at about $130^{\circ} \mathrm{C}$. We failed to determine definitely the small GPC peak found in this extrudate, but the peak may be due to the highly oriented, amorphous chain bundles in the interfibrillar region of the extruded polyethylene.

\section{REFERENCES}

1. K. Imada, T. Yamamoto, K. Shigematsu, and M. Takayanagi, J. Mater. Sci., 6, 537 (1971).

2. K. Nakayama and H. Kanetsuha, J. Mater. Sci., 10, 1105 (1975).

3. A. Peterlin, Colloid Polym. Sci., 253, 809 (1975).

4. K. Nakayama and H. Kanetsuna, J. Mater. Sci., 12, 1477 (1977).

5. Y. Maeda, K. Nakayama, and H. Kanetsuna, Polym. J., 14, 295 (1982).

6. Y. Maeda and H. Kanetsuna, Polym. J., 13, 357 (1981).

7. L. E. Nielson, J. Appl. Phys., 25, 1209 (1954).

8. M. G. Gubler and A. J. Kovacs, J. Polym. Sci., 35, 551 (1959).

9. P. R. Swan, J. Polym. Sci., 56, 403 (1962).

10. F. C. Frank, I. M. Ward, and T. Williams, J. Polym. Sci., A-2, 6, 1357 (1968).

11. T. Williams, Y. Udagawa, A. Keller, and I. M. Ward, J. Polym. Sci., A-2, 8, 35 (1970).

12. J. Clements, R. Jakeways, and I. M. Ward, Polymer, 19, 639 (1978).

13. G. Meinel and A. Peterlin, J. Polym. Sci. A-2, 6, 587 (1968).

14. A. G. Gibson, G. R. Davies, and I. M. Ward, Polymer, 19, 683 (1978).

15. M. Takayanagi, K. Imada, and T. Kajiyama, $J$. Polym. Sci., C, 15, 263 (1966)

16. G. Capaccio and I. M. Ward, J. Polym. Sci., Polym. Phys. Ed., 19, 667 (1981).

17. N. E. Weeks, S. Mori, and R. S. Porter, J. Polym. Sci., Polym. Phys. Ed., 13, 2031 (1975). 